\title{
Variations
}

Variations

Revue internationale de théorie critique

$20 \mid 2017$

Expériences oppositionnelles

\section{Tourner la page}

Éditorial

\section{OpenEdition}

Journals

Édition électronique

URL : http://journals.openedition.org/variations/873

DOI : 10.4000/variations. 873

ISSN : 1968-3960

\section{Éditeur}

Les amis de Variations

Référence électronique

"Tourner la page», Variations [En ligne], 20 | 2017, mis en ligne le 25 avril 2017, consulté le 25 février 2021. URL : http://journals.openedition.org/variations/873 ; DOI : https://doi.org/10.4000/variations. 873

Ce document a été généré automatiquement le 25 février 2021.

Les ami•e•s de Variations 


\section{Tourner la page}

\section{Éditorial}

1 Voici venu le numéro vingt de la revue Variations. Depuis près d'un an déjà, le comité a réfléchi et travaillé la question de savoir comment saisir les mouvements démocratiques et critiques qui cherchent à sortir des représentations traditionnelles des sociétés bourgeoises en Occident. À l'approche des élections présidentielles, cette problématique a pris la forme définitive d'un dossier consacré aux Expériences oppositionnelles, coordonné par Paola Sedda qui en présente les traits dans un texte introductif.

2 Le présent numéro de la revue est publié le 8 mai, une date qui porte une signification antifasciste évidente et bien connue ; elle vient aussi sonner la charge à l'encontre du programme néocapitaliste du pouvoir qui se met en place en France, au lendemain des élections. L'opposition et la résistance, dès la première heure, viendront nourrir des élans créatifs et la formation d'un vaste espace public oppositionnel dans lequel la revue s'inscrit pleinement.

Pour l'heure, les sociétés du capitalisme tardif sont contestées par des mouvements de gauche, en Europe et dans les Amériques notamment, mais elles restent dominées par un double versant autoritaire : d'une part ce mainstream capitaliste et néoconservateur qui nous est présenté comme néolibéral, d'autre part des mobilisations nationalistes, racistes et fascistes qui prospèrent sur les ruines du principe d'égalité que le premier versant a abandonné. Les élections françaises en témoignent. Notre position intellectuelle ne se laisse pas enfermer dans ce dilemme qui fut pointé par Max Horkheimer, co-fondateur de la Théorie critique, sous la forme de cet avertissement publié en 1939 : «Ceux qui ne veulent pas interroger le capitalisme doivent se taire sur le fascisme" (Wer aber vom Kapitalismus nicht reden will, sollte auch vom Faschismus schweigen). ${ }^{1}$ Nous n'allons pas nous laisser happer par ce faux choix qui consiste à évaluer les dégâts respectifs de la bureaucratie capitaliste ou de la mobilisation fasciste. Les deux s'entendent à merveille, à la fin, comme l'histoire le montre.

4 Penser la société d'aujourd'hui est une tâche ardue qui implique une prise de distance de tous les instants par rapport aux habitudes de la pensée médiatisée, toujours à l'affut de la conjoncture et de ceux qui font l'opinion, pour reprendre une formule lumineuse 
de Jean-Marie Vincent qui constitue les premières lignes du numéro 1 de Variations. ${ }^{2}$ Nous n'allons pas vivre et penser comme des porcs.

5 Notre revue, qui s'intéresse aux théories critiques à l'échelle internationale, en tant que ferment d'un projet d'émancipation global, avait compris et anticipé le choix de Jürgen Habermas qui s'est politiquement engagé aux côtés d'Emmanuel Macron, à l'encontre du socialisme historique, avant même les élections présidentielles. Le philosophe allemand applique ce programme en toute logique, après avoir soutenu le droit des propriétaires que défendit John Locke, contre le droit naturel égalitaire et révolutionnaire de Jean-Jacques Rousseau, après avoir retenu le moralisme étatique d'Émile Durkheim contre la critique de l'État de Karl Marx, et après avoir choisi la Constitution européenne de Jacques Chirac et Giscard d'Estaing plutôt que l'héritage internationaliste de Walter Benjamin. Faut-il mentionner qu'Oskar Negt, membre de notre comité international, avait déjà publié un important ouvrage collectif pour répliquer à Habermas en 1968 qui porte le titre : La gauche répond à Habermas ${ }^{3}$ ?

6 Fidèle à la démocratie socialiste qu'elle défend explicitement, Nancy Fraser - qui publie régulièrement dans nos colonnes - vient de soutenir publiquement l'opposition de gauche, en France comme aux États Unis. Avec d'autres, elle trace une perspective nouvelle qui pousse la gauche internationale à sortir définitivement de son alliance historique avec le néolibéralisme, telle qu'elle fut pratiquée par les Clinton et la socialdémocratie européenne. Elle propose de rompre avec la politique de l'identité et son discours de la diversité qui fut porté par ces courants dans le monde occidental : «À la place d'une conception anti-hiérarchique, et égalitaire de l'émancipation, consciente des rapports de classe, nous avons eu une conception individualiste, libérale de gauche. Cela a promu un groupe dominant organisé autour du principe "the winner takes it all", qui a favorisé l'ascension sociale d'un petit nombre de femmes ou d'homosexuels "particulièrement talentueux". Pendant ce temps, la majorité devait continuer à vivre à l'ombre. » Judith Butler ne tranche encore pas cette question, mais suggère désormais de relire Karl Marx et constate que l'analyse de classe a été manifestement négligée dans une partie des courants critiques. Un diagnostic que ne contestera pas Angela Davis, qui a rappelé à quel point les enseignements reçus par Theodor W. Adorno et Oskar Negt, qui fut son co-directeur de recherche, restent marquants pour elle ${ }^{4}$. Tout se passe comme si la politique de l'identité avait buté sur des limites historiques et disciplinaires qui nécessitent la relance puissante d'une Théorie critique globale.

7 Au moment du bouclage, nous avons appris le décès de Miguel Abensour, sociologue et philosophe politique qui a en partie formé des membres de notre revue, dont il fut par ailleurs l'un des contributeurs. Il nous laisse une œuvre précieuse, après avoir fait connaître, dans l'espace francophone, des auteurs incontournables comme Max Horkheimer, Siegfried Kracauer ou Franz Neumann. Nous lui rendons hommage.

En Hors-champ, nous proposons des articles de Fabien Granjon et Simon Le Roulley, consacrés à des aspects actuels des pensées d'Axel Honneth et d'Henri Lefebvre.

9 Le numéro 21 de la revue, qui est envisagé pour l'hiver 2017, aura pour enjeu l'actualisation de l'industrie de la culture en tant que concept critique, en écho à sa formulation originale par Adorno et Horkheimer en 1942. Il réunira des chercheuses et chercheurs francophones, germanophones et anglophones, dont Fredric Jameson et Oskar Negt. 


\section{NOTES}

1. M. Horkheimer, « Die Juden und Europa » in: Gesammelte Werke, vol.4, Suhrkamp, 1988, p.308.

2. J.-M. Vincent, « Variations? » in : Variations n.1, Syllepse, 2001, p.7.

3. O.Negt, Die Linke antwortet Habermas, EVA, 1968.

4. Voir les entretiens de presse suivants : J. Habermas, Le Monde, 19/4/2017; N.Fraser, Taz, Berlin, 2/5/2017; J. Butler, ND, Berlin, 29/4/2017; A. Davis, Taz, Berlin, 5.12.2013. 